\title{
Investigating dynamic pain sensitivity in the context of the fear-avoidance model
}

\author{
C.W. Gay ${ }^{1}$, M.E. Horn ${ }^{1}$, M.D. Bishop ${ }^{2}$, M.E. Robinson ${ }^{3}$, J.E. Bialosky ${ }^{4}$ \\ 1 Rehabilitation Science Doctoral Program, University of Florida, Gainesville, USA \\ 2 Department of Physical Therapy, University of Florida, Gainesville, USA \\ 3 Department of Clinical and Health Psychology, University of Florida, Gainesville, USA \\ 4 Department of Physical Therapy, University of Florida, Gainesville, USA
}

\author{
Correspondence \\ Charles W. Gay \\ E-mail: chaz.gay@phhp.ufl.edu \\ Funding sources \\ This manuscript was written while C.W.G. \\ received support from the University of \\ Florida Alumni Fellowship and NCMIC \\ Foundation, M.D.B. and M.E.H. received \\ support from the National Center of \\ Complementary and Alternative Medicine \\ (R01AT006334). This study was funded by a \\ University of Florida Faculty Opportunity Fund \\ awarded to J.E.B.
}

Conflicts of interest

None declared.

Accepted for publication

6 April 2014

doi:10.1002/ejp.519

\begin{abstract}
Background: Although nearly everyone at some point in their lives experiences back pain; the amount of interference with routine activity varies significantly. The fear-avoidance (FA) model of chronic pain explains how psychological variables, such as fear, act as mediating factors influencing the relationship between clinical pain intensity and the amount of interference with daily activities. What remains less clear is how other mediating factors fit within this model. The primary objective of this report was to examine the extent to which a dynamic measure of pain sensitivity provides additional information within the context of the FA model.

Method: To address our primary objective, classic mediation and moderated mediation analyses were conducted on baseline clinical, psychological and quantitative sensory measures obtained on 67 subjects with back pain (mean age, $31.4 \pm 12.1$ years; $70 \%$ female).

Results: There was a moderately strong relationship $(r=0.52 ; p<0.01)$ between clinical pain intensity and interference, explaining about $27 \%$ of the variance in the outcome. Mediation analyses confirmed fear partially mediated the total effect of clinical pain intensity on interference $(\Delta \beta=0.27 ; p<0.01)$, and accounted for an additional $16 \%$ of the variance. In our FA model, pain sensitivity did not demonstrate additional indirect effects; however, it did moderate the strength of indirect effects of fear.

Conclusion: This preliminary modelling suggests complex interactions exist between pain-related fear and pain sensitivity measures that further explain individual differences in behaviour.
\end{abstract}

\section{Introduction}

The lifetime prevalence of low back pain (LBP) ranges from $11 \%$ to $84 \%$ of all adults (Walker, 2000; Deyo et al., 2006), depending on the definition of an episode. The overall impact of LBP is substantial, but there is significant individual variability on how LBP influences daily activities. Only $51 \%$ of the individuals who report LBP in the past 3 months also report difficulty with simple basic actions, while LBP creates difficulty in complex activities in only $55 \%$ of indi- viduals (Institute of Medicine (IOM), 2011). Further, the relationship between the intensity of LBP and the amount of pain-related interference in performing daily activities is not uniform (Von Korff et al., 1992).

A traditional biomedical approach to explain nonuniformity may focus on structural abnormalities of the spine suggesting differences in the severity and type of the injury account for differences in pain intensity and related interference. However, studies have found that structural pathology of the spine is only weakly, if at all, associated with individual 


\section{What's already known about this topic?}

- Psychological variables are important intermediate factors between clinical pain and disability.

- Quantitative sensory testing (QST) measures are predictive of clinical pain intensity and can discriminate healthy controls from patients with low back pain with QST scores suggesting the presence of pain hypersensitivity.

\section{What does this study add?}

- This study attempts to model interactions between psychological and QST measures simultaneously, with the fear-avoidance model of musculoskeletal pain (FAM) as the initial starting point.

differences in clinical outcomes (Chou et al., 2011). Fortunately, pain and pain-related behaviour are no longer viewed in a purely biomedical model, but from a biopsychosocial perspective, which takes into consideration biological (such as spinal abnormalities, genetics and nervous system adaptations), psychological (such as emotions and beliefs) and societal (such as cultural norms and values and social support) factors. The purpose of this paper is to explore factors influencing the relationship between intensity of LBP and pain-related interference with standard activities. Specifically, we will examine how two factors, fear of pain experienced during standard activities and dynamic pain sensitivity, influence this relationship.

We chose fear of pain because we feel it is representative of constructs within the fear-avoidance (FA) model of chronic pain. The FA model of chronic pain illustrates how individual differences in psychological variables explain individual variability on how pain impacts behaviour and disability. The FA model has been applied to several chronic pain populations including chronic LBP, and other pain populations such as acute LBP and experimental pain (Fritz et al., 2001; Leeuw et al., 2007; George et al., 2008; George and Hirsh, 2009; Crombez et al., 2012; Parr et al., 2012). Because the FA model has gained such support over the years, we will first model the influence of our psychological factor on the relationship between intensity of LBP and pain-related interference. The innovative aspect of this study is that we will then concurrently investigate the influence of another intermediate factor, dynamic pain sensitivity.

Pain sensitivity testing is suitable for investigating individual differences in the pain processing system that encodes the experience from stimuli originating from the body. There are many pain sensitivity protocols currently being employed in research. Research has demonstrated that certain pain sensitivity protocols (1) can adequately discriminate populations with and without pain; (2) are modestly associated with clinical pain intensity; and (3) are modestly associated with physical disability. For discriminating populations, Neziri et al., 2012, found that 6 out of 26 pain sensitivity protocols assessed adequately discriminated healthy controls from patients with chronic LBP (Neziri et al., 2012). Those six pain sensitivity tests were pressure pain thresholds in the area of pain, electrical pain thresholds, electrical stimulation reflex threshold, pressure pain tolerance in the area of pain, pressure pain threshold in a remote location and temporal summation of pain (TSP) threshold (Neziri et al., 2012).

For the association between clinical measures and pain sensitivity measures, Hubscher et al., 2013 performed a systematic review of the literature and pooled the estimated correlation between four pain sensitivity protocols and the intensity of clinical pain and between two pain sensitivity protocols and clinical disability in people with spinal pain (Hubscher et al., 2013). They found pain intensity was weakly associated with the pain sensitivity protocols of pain threshold $[-0.15,95 \%$ confidence interval (CI): -0.18 to $-0.11]$, pain tolerance $(-0.30,95 \% \mathrm{CI}:-0.45$ to $-0.13)$ and TSP $(0.16,95 \%$ CI: 0.02 to 0.29$)$ but not associated with pain magnitude of a suprathreshold stimulus $(0.25,95 \%$ CI: -0.16 to 0.66$)$. They also found that pain-related disability was weakly associated with pain thresholds $(-0.16,95 \% \mathrm{CI}:-0.22$ to $-0.10)$ but not pain tolerance $(-0.32,95 \%$ CI: -0.69 to 0.17; Hubscher et al., 2013). Another recent study, not included in the review, found the magnitude of pain intensity reported to specific amounts of pressure and thermal stimuli was highly predictive of clinical pain intensity in subjects with musculoskeletal pain, explaining approximately $38 \%$ and $26.8 \%$ of the variance, respectively (Staud et al., 2012).

Recently, potential predictors of pain-related behaviour have expanded beyond just clinical pain intensity to include factors from different aspects of the biopsychosocial realm. For example, Alschuler et al. included, pain intensity as well as a measure of psychology, pain sensitivity and social support. They found the static measure of pain sensitivity (pressure pain thresholds) explained individual variation in physical activity among chronic LBP subjects, after accounting for pain intensity, fear avoidance and solicitous spousal responses (Alschuler et al., 2011). This line of investigation is encouraging; however, 
gaps in understanding persist because of the numerous pain sensitivity protocols being used in research, especially dynamic pain sensitivity protocols.

Dynamic pain sensitivity protocols, such as TSP, are techniques which are frequently used to study endogenous pain modulatory mechanisms. TSP, in particular, assesses the change in perceived pain intensity to repeated stimulation, where amplification of pain perception is often seen following repeated stimuli. (Arendt-Nielsen and Yarnitsky, 2009). Research has shown that individuals with chronic pain conditions often shown increased amplification of pain perception when compared with individuals without pain. Despite the average increase in pain amplification within chronic pain populations, responses to a TSP protocol remain variable within given cohorts of individuals (Anderson et al., 2013). Unlike the static pain sensitivity protocol used in the Alschuler et al. study, the clinical implications for TSP has not been thoroughly investigated within a biopsychosocial model. Very little has been published except for the George et al. study in 2006. That study found variability in TSP explained variation in perceived disability among chronic LBP subjects, after accounting for fear avoidance and clinical pain (George et al., 2006).

In this study, we chose to investigate the role of TSP within the context of the FA model. This approach is compatible with current suggestions to expand the FA model. Suggestions for expansion included a role of stimuli, originating from the body, working as a conditioning prompt (Vlaeyen and Linton, 2012). In our approach, first, we will model the direct effects of clinical pain intensity on pain-related interference going through an intermediate psychological factor. From there, we will investigate whether the responsiveness of the nervous system, assessed with dynamic pain modulation protocol (i.e., TSP) acts as an additional mediator or moderator. A significant moderated mediation model would suggest the indirect effects of pain-related fears in predicting the level of daily interference are conditional, depending on the level on pain sensitivity. Therefore, our purpose in this paper was to build and test mediation and moderated mediation models using baseline clinical, psychological and pain sensitivity measures in a cohort of individuals with non-specific LBP.

\section{Materials and methods}

\subsection{Overview}

Analyses were performed on baseline clinical, psychological and pain sensitivity measures collected from the first 80 individuals enrolled in a randomized clinical trial (ClinicalTrials.gov Identifier: NCT0 1 168999) comparing manual therapy techniques for LBP (Bialosky et al., 2014). The study received approval by the University of Florida Institutional Review Board. All participants provided informed consent. Baseline measurements were obtained prior to randomization.

\subsection{Participants}

Participants were recruited from the general community through posted study announcements and word of mouth. Participants were eligible to participate in the study if they were currently experiencing LBP that did not extend below the knee, rated their worst LBP over the past $24 \mathrm{~h}$ as a minimum of 4 out of $10(0=$ no pain, $10=$ worst pain imaginable), were appropriate for conservative management of LBP and spoke English. Individuals were excluded if they had surgery to the low back in the past 6 months, were diagnosed with a systemic disease known to effect sensation, other chronic pain condition unrelated to the LBP or reported the cause of their LBP to be a fracture.

\subsection{Baseline clinical measures}

\subsubsection{Clinical pain intensity}

LBP intensity was assessed using the Patient-Centered Outcomes Questionnaire (PCOQ; Brown et al., 2008). Subjects responded to the statement 'First, we would like to know your usual levels of pain, on a scale of 0 (none) to 100 (worst imaginable), please indicate your usual level (during the past week) of pain' (Brown et al., 2008). We operationally defined this value to indicate each person's usual level of pain intensity associated with their LBP.

\subsubsection{Clinical pain interference}

Pain interference was assessed with the modified Oswestry Disability Index (ODI). The original version described by Fairbank et al. (1980) was modified by inserting employment/homemaking ability for the section previously related to sex life (Fairbank et al., 1980; Fairbank and Pynsent, 2000; Roland and Fairbank, 2000). The ODI has 10 items that assess how LBP affects common daily activities (e.g., sitting, standing and lifting). The ODI has a range of percentages from $0 \%$ ('no disability due to back pain') to $100 \%$ ('completely disabled due to back pain'), where higher scores indicate higher interference from LBP. The ODI has been found to have high levels of test-retest reliability [intraclass correlation $($ ICC $)=0.90]$, convergent validity with the Roland Morris disability questionnaire $(r>0.80)$ and responsiveness (effect size $=1.8$ ) in patients receiving therapy for LBP (Fairbank and Pynsent, 2000; Roland and Fairbank, 2000) and is recommended as an appropriate measure of self-report of disability for patients with LBP (Dworkin et al., 2008; Chapman et al., 2011). 


\subsection{Baseline psychological measures}

The Fear-Avoidance Beliefs Questionnaire (FABQ) was used to quantify general fear-avoidance beliefs. This questionnaire focuses on subject's beliefs about how work and physical activity effect patients' LBP. The FABQ is an 11 -item 7-point rating scale $(0$ 'strongly disagree' to 6 'agree') with physical activity (FABPA) and work (FABQW) sub-scales. Higher FABQ scores indicate higher levels of fear-avoidance beliefs and the FABQPA ranges from $0-24$ while the FABQW ranges from 0 to 42 (Waddell et al., 1993). The test-retest stability of FABQ has been reported in the literature for patients with chronic low back pain with a kappa for individuals items of 0.74 (Waddell et al., 1993).

The Pain Catastrophizing Scale (PCS) has 13 items assessing catastrophizing thoughts, where subjects use a 5-point scale ( 0 'not at all' to 4 'all the time') to rate the frequency of these thoughts. A PCS sum score was calculated for all items (range 0-52), with higher scores indicating higher frequencies of pain catastrophizing thoughts (Sullivan et al., 1995). The PCS has adequate internal consistency (Cronbach's alpha - 0.87) and good 6- and 10-week test-retest reliability $(r=0.75$ and 0.70, respectively; Sullivan et al., 1995).

The Fear of Daily Activities Questionnaire (FDAQ) was used to quantify the fear of particular activities. This questionnaire lists 10 activities that patients with LBP commonly report as being fearful of performing due to LBP (George and Hirsh, 2009; George et al., 2009). The FDAQ has two options for open-ended responses so that patients with LBP can provide additional examples and ratings of activities that they fear performing due to pain. Patients rate each FDAQ item using a NRS ranging from 0 ('no fear') to 100 ('maximal fear'). Scoring of the FDAQ adds each NRS rating from the 10 standard activities and dividing the sum by 10. Higher FDAQ scores indicate higher fear of activities. The open-ended responses were not included in scoring because responses vary. The FDAQ demonstrated adequate internal consistency [Cronbach's alpha $0.91 ; 95 \%$ CI $(0.87,0.95)$ ] and 48 h testretest properties [ICC coefficient $0.90,95 \%$ CI $(0.82,0.94)$ ] (George and Hirsh, 2009; George et al., 2009). The minimal detectable change of the FDAQ based on the standard error of measurement of 6.6 is 12.9 (George and Hirsh, 2009; George et al., 2009).

\subsection{Baseline pain sensitivity measures}

Thermal TSP was used to quantify endogenous pain modulatory mechanisms. TSP is a dynamic pain sensitivity measure of pain facilitation (e.g., amplification). This measure focuses on the change in perception over time to repeated stimuli. There are similarities between TSP, wind-up and central sensitization. Wind-up is an experimental method of inducing N-methyl-D-aspartate (NMDA) receptor mediated neuronal plasticity that manifests as an increase in the excitability of dorsal horn spinal neurons (Todd, 2010). It is generated by applying a train of repetitive stimulation of nociceptive fibres, delivered at a constant intensity of $0.3 \mathrm{~Hz}$ or greater, causing the spinal neuronal excitability to increase cumulatively with each successive stimulus resulting in amplification (e.g., facilitation) of the signal. Changes within the dorsal horn (i.e., central sensitization) are thought to contribute to amplification of nociception after injury and inflammation and provide the physiological basis for primary and secondary hyperalgesia and allodynia (Latremoliere and Woolf, 2009; Todd, 2010). The correlate of wind-up in humans is thought to be perceptual wind-up, commonly referred to as TSP (Staud et al., 2006). This is because TSP is described as increases in pain perception to a train of stimuli of the same intensity delivered at $0.3 \mathrm{~Hz}$ or greater and is also readily attenuated by NMDA receptor blockade (Sarkar et al., 2006; Zhou et al., $2011)$. TSP is considered a global measure of amplification within the sensory and perceptual systems and is not specifically restricted to the spinal cord.

Thermal stimuli were generated using the Medoc Neurosensory Analyzer TSA II (TSA-2001, Ramat Yishai, Israel). A train of 10 heat pulses delivered at a frequency $<0.33 \mathrm{~Hz}$ was applied to a remote location outside of the primary area of complaint (i.e., lower back) to avoid confounds of changes to the peripheral nervous system (Arendt-Nielsen and Yarnitsky, 2009). The thermode was placed on the plantar surface of each subject's dominant foot, and the temperature rapidly fluctuated $\left(10^{\circ} \mathrm{C} / \mathrm{s}\right)$ from $39^{\circ} \mathrm{C}$ to a peak of $50{ }^{\circ} \mathrm{C}$, remained at peak temperature for $<\mathrm{ls}$, and returned to baseline by active cooling. Subjects were asked to rate the intensity of pain associated with each pulse using a numeric rating scale (NRS) anchored with $0=$ 'no pain' to $100=$ 'the worst pain imaginable'. In cases where an NRS response for a particular pulse was not recorded, the data was considered missing and the individual was removed from the analysis due to incomplete data.

Several methods have been used to calculate the magnitude of TSP; however, agreement on which method is most acceptable and clinically relevant has not been determined (Anderson et al., 2013). Therefore, we initially used four methods. Three of those methods have been previously reported, which included (1) the NRS rating of the 5th pulse, (2) a subtraction method using the NRS rating of the 5th pulse minus the NRS rating of the 1st pulse ( 5 minus 1 ) and (3) a simple slope, which calculates the average change in NRS responses over each of the pulses (Bialosky et al., 2009; Bishop et al., 2011; Valencia et al., 2011). A fourth, novel method was also included. This method uses the area under the response curve generated by the 10 NRS pain intensity ratings. This novel method, complete area under the curve, considers both the initial pain rating and the temporal characteristics of change in pain ratings over time. These variables met normality assumptions and were treated as continuous in the subsequent analyses. TSP estimates, calculated with the simple slope method described herein, have been shown to be moderately stable when repeated within a single testing session for both pain-free and induced acute pain participants $[\mathrm{ICC}=0.58 ; 95 \% \mathrm{CI}=(0.47,0.67)$ and 
ICC $=0.49 ; \quad 95 \% \quad$ CI $=(0.29,0.76)$ respectively $\quad$ (Alappattu et al., 2011). TSP, estimated with the same method, assessed in pain-free subjects, was shown to be less stable across two-testing sessions that were 2 weeks apart [ICC $=0.37$; $95 \% \mathrm{CI}=(0.01,0.66)]$ (Alappattu et al., 2011$)$.

\section{Data Analyses}

Analyses were performed using IBM SPSS Statistics version 20.0 (SPSS Inc., Chicago, IL, USA). Significance levels were set at an alpha level of 0.05 for analyses. Cases with incomplete data were removed using list-wise deletion. Descriptive analyses of the sample were generated and reported for all variables in the appropriate metric. Histograms were used to visually inspect the distribution of each variable and tests of normality (i.e., Kolmogorov-Smirnov and Shapiro-Wilks) were conducted prior to hypothesis testing. Bivariate correlations were calculated to assess baseline relationships among all clinical, psychological and pain sensitivity variables

In our analyses, assumptions from the FA model were employed; such that pain-related fears were used as the mediator (indirect effect) in the relationship between clinical pain intensity and self-reported pain interference with daily activities, see Fig. 1A. To explore what effect an individual's TSP response has on has on this model, we assessed whether pain sensitivity qualified as a separate mediator, or moderated the indirect effects of fear in the model; see Fig. 1B. Traditional mediation and moderated mediation analysis techniques, developed by Baron and Kenny and refined by Preacher and Hayes, were employed (Baron and Kenny, 1986; Preacher and Hayes, 2004).

\subsection{Mediation}

Mediation analyses were conducted using the PROCESS macro for SPSS provided by Hayes, (Hayes, 2012). PROCESS uses ordinary least squares regression to generate direct and indirect effects in mediation models and conditional direct and indirect effects in moderated mediation models. To test our hypotheses, we used two theoretical PROCESS Models, 4 and 58. PROCESS Model 4 tests for simple mediation (i.e., indirect effects) and allows for up to 10 mediators to be acting in parallel (see Fig. 1A). PROCESS Model 58 tests for conditional indirect effects of both the ' $a$ ' and 'b' paths (see Fig. 1B). Prerequisite criteria were first established prior to testing for mediation. The three criteria were (1) the mediator needed to be correlated with the independent variable, (2) the outcome variable needed to be correlated with the independent
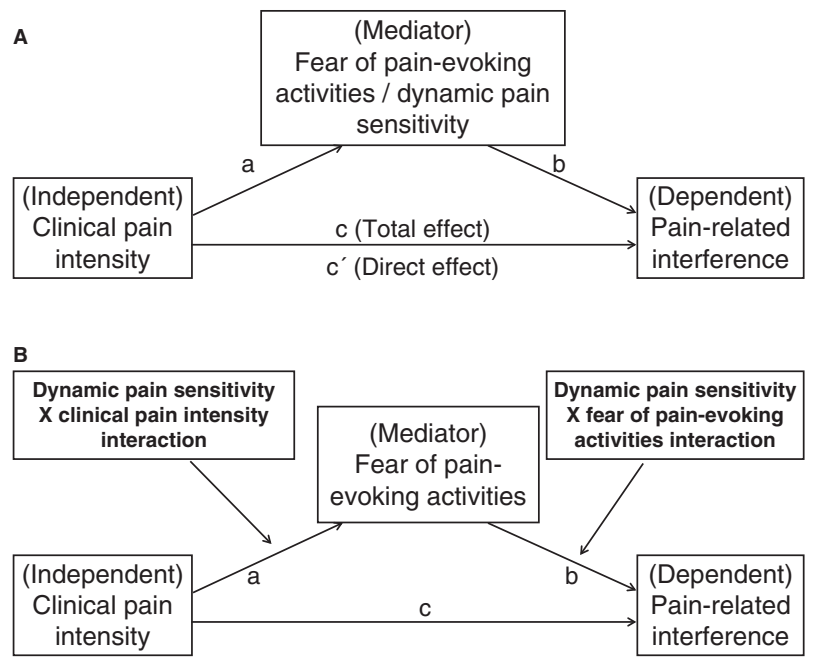

Figure 1 Presumptive models. (A) Mediation model. (B) Moderatedmediation model. (A) Mediation model assumes low back pain intensity has a significant total effect on pain-interference, [Total Effect of $X$ on $Y=(c)$ ]. Consistent with the Fear Avoidance model, pain-related fears are expected to mediate this relationship, [Indirect effects of $X$ on $Y$ through $M=(a) \times(b)]$. The direct effect represents the effect of pain intensity on pain interference after the indirect effects are removed [Total Effect Indirect Effects $=$ Direct Effect $=(\mathrm{C})$ ]. Whether pain sensitivity is an additional mediator will be tested. (B) Moderated Mediation model assumes pain-related fears mediate the relationship between low back pain intensity and pain-interference. This model tests whether the indirect effects through pain-related fears are conditional based on the level of pain sensitivity. Conditional indirect effects are tested for both the first and second stage, path $a$ and $b$ respectively.

variable and (3) the mediator needed to be correlated with the outcome variable. If pre-conditions were met, separate linear regression models were used to calculate the indirect effects (i.e., mediation), for painrelated fears and pain sensitivity. Sobel's test was used to test the significance of the indirect effects (Preacher and Hayes, 2004). In the event prerequisite conditions were met and significant indirect effects were found for both factors, a combined regression model with both mediating factors acting in parallel would be conducted using 'model 4' of the PROCESS macro.

\subsection{Moderated mediation analysis}

Moderated mediation analyses were conducted using the methods described by Preacher et al. (2007) and 'model 58' of the PROCESS macro for SPSS provided by Hayes (2012). PROCESS model 58 tests for conditional indirect effects of both the ' $a$ ' and ' $b$ ' paths (see Fig. 1B). Hierarchical regression procedures were conducted to test whether the indirect effects of painrelated fears are conditional depending on the level of 
pain sensitivity. Separate regression procedures were conducted for the first stage (path a) and second stage (path b). Conditional indirect effects are represented by a significant interaction. Interaction terms were computed by multiplying mean-centred variables. Simple slope analyses and the Johnson-Neyman technique were used to decompose significant interactions. For simple slope analyses, high, intermediate and low values of the moderator (pain sensitivity) corresponded to 1 standard deviation (SD) above the mean, the mean, and I SD below the mean, respectively. The Johnson-Neyman technique estimates the value of the moderator where the indirect effects of mediator are significantly different at an alpha of 0.01 (Butsch, 1994; Hayes and Matthes, 2009).

\section{Results}

Of the 80 subjects, 67 had complete data required for the proposed analysis. Thirteen subjects were excluded from further analyses because of missing FDAQ (6), incomplete TSP data (5) or incomplete ODI assessment (2). Independent samples $t$-tests showed patients that were excluded from further analysis were not significantly different in age, gender, level of disability, usual pain intensity, FDAQ, PCS, FABQ or TSP from the remainder of the sample. Of the 67 subjects, $47(70 \%)$ were female and a mean age of 31.42 years $(\mathrm{SD}=12.06)$. The mean LBP intensity, 'usual level over the past week', of the cohort was 42.71 $(\mathrm{SD}=23.19)$ and the mean pain interference, (ODI) was $17.30 \%(\mathrm{SD}=11.75 \%)$. The mean duration of pain was 203.91 weeks $(S D=377.78)$; see Table 1. Visual inspection of histograms and normality tests suggest all the variables approximate a normal distribution and were appropriate for our planned correlation and regression analyses.

\subsection{Baseline correlations}

LBP intensity was positively and significantly correlated with self-reported pain-related interference, $(r=0.52 ; p<0.01)$; see Table 1. Most psychological measures were positively and significantly correlated with both LBP intensity and pain-related interference. These measures were also positively correlated with one another. Fear of specific daily activities, (FDAQ) had the largest point estimates of association with both LBP intensity and pain-related interference, $(r=0.56$; $p<0.01$ and $r=0.63 ; p<0.01$, respectively). Dynamic pain sensitivity appeared to be weakly and positively associated clinical pain intensity for all methods of calculating TSP, but none of these estimates were sig-

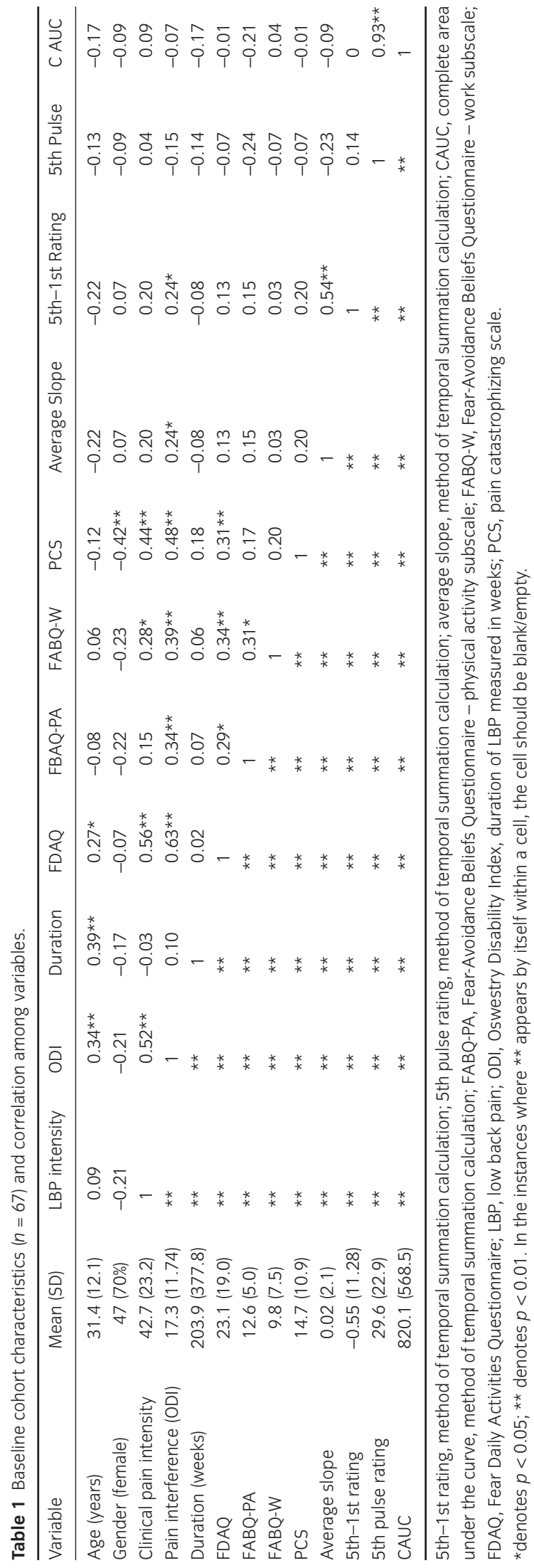




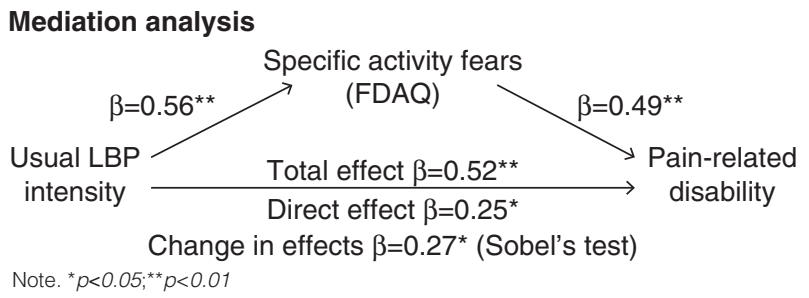

Figure 2 Mediation analysis. Consistent with the Fear Avoidance model, pain intensity had a significant total effect on pain-interference (c). Painrelated fears mediated this relationship, [Indirect effects of $X$ on $Y$ through $M=(a) \times(b)$ ]. The direct effect of pain intensity on pain interference after the indirect effects are of pain-related fears was removed remained significant, suggesting partial mediation. $\beta=$ standardized beta coefficients; FDAQ $=$ Fear of Daily Activities Questionnaire; LBP = low back pain.

nificant. Two of the TSP methods of calculation were found to be weakly and positively correlated with pain-related interference, 'average slope' and ' 5 minus $l^{\prime}$. The other two methods of calculation were weakly and negatively associated with pain-related interference; however, neither of these estimates were significant. We did not find significant or consistent direction of correlation between psychological factors and pain sensitivity. Across the four methods of calculating TSP, 'average slope' and ' 5 minus 1 ' were moderately correlated $(0.54, p<0.01)$ with one another. While 'magnitude of 5th pulse' and 'complete area under the curve' were strongly correlated with one another $(r=0.93 ; p<0.01)$.

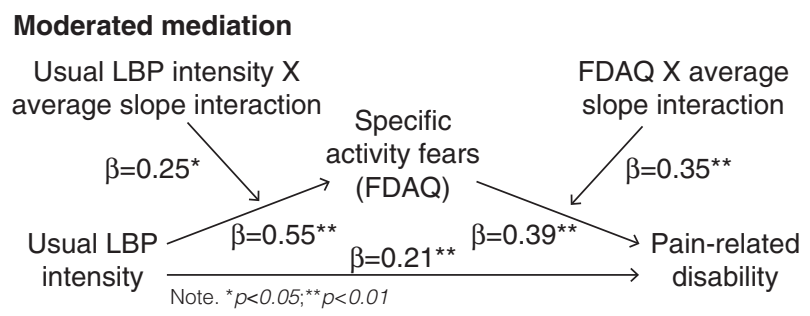

Figure 3 Moderated mediation. Significant interactions were found for both the first and second stage of the mediation model, suggesting the indirect effects through pain-related fears are conditional based on the level of pain sensitivity. $\beta=$ standardized beta coefficients; FDAQ = Fear of Daily Activity Questionnaire; LBP = low back pain; Average slope = temporal summation of pain.

FDAQ and average slope had the highest estimated zero-order correlations with the clinical measures of pain intensity and pain-related interference. Therefore, we chose FDAQ to represent the psychological measure of fear in the mediation and moderated mediation analyses and average slope represented the dynamic pain sensitivity measure.

\subsection{Mediation}

Prerequisite criteria for mediation were established for pain-related fears (FDAQ), but not pain sensitivity (TSP). Therefore, mediation was only investigated for pain-related fears. The overall model, with LBP inten-

Table 2 Moderated Mediation analyses: hierarchical regression testing for first- and second-stage conditional effects of pain sensitivity.

\begin{tabular}{|c|c|c|c|c|c|}
\hline Variables & Adjusted $R^{2}$ & $p$-value & B (standard error) & Standardized $\beta$ & $p$-value \\
\hline \multicolumn{6}{|c|}{ Dependent variable: specific activity-related fear (FDAQ) } \\
\hline Step 1 & 0.29 & $<0.01 *$ & & & \\
\hline Usual LBP intensity & & & $0.46(0.09)$ & 0.56 & $<0.01 *$ \\
\hline TSP & & & $0.13(0.96)$ & 0.01 & 0.13 \\
\hline Step 2 & 0.34 & $<0.01 *$ & & $\mathrm{R}^{2}$ change $=0.06$ & $p=0.02^{\star}$ \\
\hline Usual pain intensity & & & $0.45(0.08)$ & 0.55 & $<0.01 *$ \\
\hline TSP & & & $-0.61(0.98)$ & -0.07 & 0.54 \\
\hline Interaction $(\mathrm{LBP} \times \mathrm{TSP})$ & & & $0.07(0.03)$ & 0.25 & $0.02^{\star}$ \\
\hline \multicolumn{6}{|c|}{ Dependent variable: LBP-related disability (ODI) } \\
\hline Step 1 & 0.43 & $<0.01 *$ & & & \\
\hline Usual LBP intensity & & & $0.11(0.06)$ & 0.23 & $0.05^{\star}$ \\
\hline $\mathrm{FDAQ}$ & & & $0.30(0.07)$ & 0.48 & $<0.01 *$ \\
\hline TSP & & & $0.76(0.53)$ & 0.14 & 0.16 \\
\hline Usual LBP intensity & & & $0.11(0.05)$ & 0.21 & $0.04 *$ \\
\hline $\mathrm{FDAQ}$ & & & $0.24(0.07)$ & 0.39 & $<0.01 *$ \\
\hline TSP & & & $-0.02(0.54)$ & -0.01 & 0.97 \\
\hline Interaction (FDAQ × TSP) & & & $0.07(0.02)$ & 0.35 & $<0.01 *$ \\
\hline
\end{tabular}

$\beta$, standardized parameter estimate; $B$, raw parameter estimate; LBP, low back pain; TSP, temporal summation of pain using average slope method of calculation.

$\star$ denotes $p<0.05$. 
sity and pain-related fears as predictors, explained $42 \%$ of the variation in pain-related interference (adjusted $R^{2}=0.42 ; F_{(2,64)}=24.70 ; p<0.01$ ). Consistent with the FA model, the direct effects of LBP intensity $(\beta=0.52 ; p<0.01)$ on pain-related interference was significantly reduced, $(\Delta \beta=0.27$; Sobel's $t=3.37, p<0.05)$ suggesting pain-related fear partially mediates pain intensity's effect on interference; see Fig. 2.

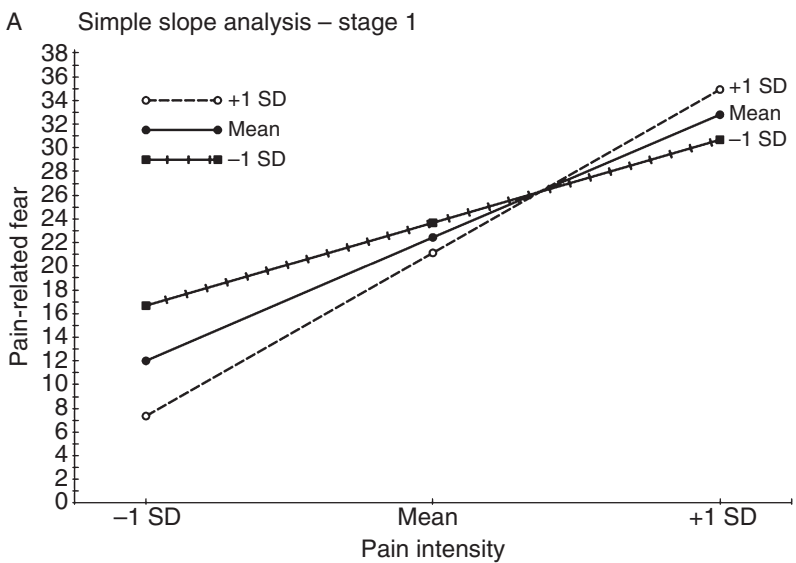

B

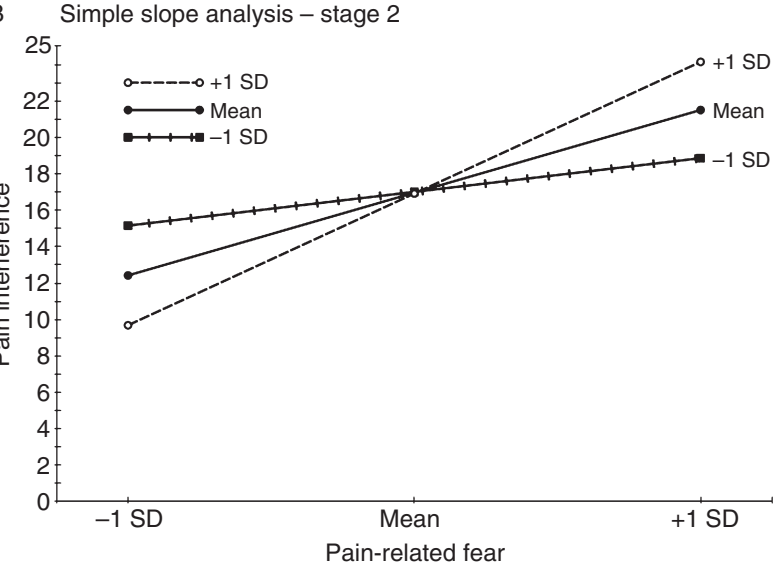

Figure 4 Simple slope analyses. (a) Simple slope analysis - Stage one. (b) Simple slope analysis - Stage two. (A) Simple slope analysis for stage one. Results suggests that as pain intensity increases the predicted level of pain-related fears to specific daily activities increases at a higher rate for higher pain sensitivity compared to lower pain sensitivity. (B) Simple slope analysis for stage two. Results suggests that as pain-related fears of specific activities increases the predicted level of pain-interference increases at a higher rate for higher pain sensitivity compared to lower pain sensitivity. Stage two analysis statistically controlled for pain intensity. Painrelated fear = fear of daily activity; Pain intensity = usual low back pain intensity; Pain interference $=$ Oswestry Disability Questionnaire; Mean = mean score; -1 SD $=1$ standard deviation below the mean; +1 $\mathrm{SD}=1$ standard deviation above the mean; TSP = temporal summation of pain, using average slope method to calculate score.

\subsection{Moderated mediation}

Pain sensitivity moderated both the first- and secondstage indirect effects; see Fig. 3. Hierarchical regression analyses found significant pain sensitivity by pain intensity (adjusted $R^{2}=0.34 ; F_{(3,63)}=12.40 ; p<0.01$; $\left.\beta=0.25 ; t=2.36 ; p<0.05 ; \Delta R^{2}=0.06 ; p<0.05\right)$ and pain sensitivity by pain-related fear interactions, (adjusted $R^{2}=0.51 ; F_{(4,62)}=18.30 ; p<0.01 ; \beta=0.35$; $t=3.5 ; p<0.01 ; \Delta R^{2}=0.09 ; p<0.01$ ) suggesting conditional indirect effects; see Table 2 . For stage 1 , as pain intensity increases, the predicted amount of painrelated fears increases at a higher rate for higher levels of pain sensitivity compared with lower levels. This pattern was similar for stage 2 . As the level of painrelated fears increases, the predicted amount of painrelated interference increases at a higher rate for higher levels of pain sensitivity. Fig. 4A and B illustrate the results of the simple slopes analysis, depicting the regression lines at high, medium and low (+1 SD, mean, $-1 \mathrm{SD}$ ) values of pain sensitivity. Results of the Johnson-Neyman analysis for stage 1 found that the effects of pain intensity on pain-related fears transitioned at pain sensitivity scores of $-1.57 ; \beta=0.34$; $\mathrm{SE}=0.10 ; t=3.46 ; p<0.01 ; 95 \%$ CI $(0.14,0.53)$. For stage 2, Johnson-Neyman analysis found that the effects of pain-related fears on pain-related interference transitioned at pain sensitivity scores of -0.24 ; $\beta=0.22$; SE $=0.07 ; t=3.28 ; p<0.01 ; 95 \%$ CI $(0.09$, 0.36). Approximately $60 \%$ of the sample reported a score of -0.24 or higher.

\section{Discussion}

The baseline correlations among the various clinical, psychological and pain sensitivity measures are in line with previous research. Our results for the dynamic pain sensitivity measure were similar to those reported by Hubscher et al., 2013, where a weak relationship between TSP and clinical pain intensity was found. Our point estimate for this correlation was slightly higher than those reported for the overall pooled estimate of $r=0.16$ (95\% CI: 0.02 to 0.29 ), and slightly lower than the pooled estimate in the sub-analysis using just chronic low back pain and thermally induced TSP $(r=0.26 ; 95 \%$ CI: 0.09 to 0.42$)$. However, our estimates were non-significant. To achieve significance with our correlation of determination $\left(R^{2}=0.04\right)$ a sample size of 191 people would have been needed. We also report a similar weak relationship between TSP and our disability measure, which was not assessed in the 2013 systematic review. Our results indicate that even though TSP might help 
discriminate between groups (pain versus pain-free), the ability of TSP to predict the intensity of pain or the severity of interference alone seems limited.

We also reported on the bivariate correlation among four different methods of calculating TSP for an individual. The two methods, average slope across all 10 pulses and the difference between the 5th pulse and 1st pulse, appear to be related one another as well as share similar relationships with the clinical measures. The other two methods, magnitude of the rating of the 5 th pulse and complete area under the curve, were highly related; however, their relationship to clinical measures appears weaker. Our data suggests that assessing the change over time using more pulses may be the most clinical relevant method of calculating an individual's TSP.

The positive correlation between the psychological measures with clinical measures and with one another is consistent with the FA model. It is likely that the FDAQ-ODI correlation was stronger than the other psychological measures because many of the items are more specific to similar constructs. For example, FDAQ asks about fear of pain while walking, sitting and standing, while the ODI inquires about how pain interferes with similar activities, such as walking, sitting and standing. The other psychological measures are more generalized. Vlaeyen and Linton (2012) have hypothesized that by virtue of pain's biological significance, it initiates both implicit and explicit learning (Vlaeyen and Linton, 2012). Through these associative learning processes, an individual's fear of pain becomes refocused onto fears of environments, activities and situations. This hypothesis is consistent with the FDAQ-ODI correlation being slightly stronger than the other psychological measures. This is likely because many of the items in both measures are similar in constructs. For example, FDAQ asks about fear of pain while walking, sitting and standing, while the ODI inquires about how pain interferes with similar activities, such as walking, sitting and standing. The other psychological measures are more generalized.

Also consistent with previous research was our mediation analysis, which showed that pain-related fears mediate the impact of pain severity upon interference (Gheldof et al., 2010). The novelty of our study is that we further showed that the level of dynamic responsiveness of the pain perception system, assessed with TSP, modulated the strength of the indirect effects of fear. Our significant stage 1 interaction between pain intensity and TSP, suggest that as the amount of LBP increases, higher TSP is associated with acquiring higher levels of pain-related fears to standard activities. Our stage 2 interaction supports that after controlling for usual pain intensity, as the amount of fear of daily activities increases, individuals with elevated TSP experience greater life interference due to pain than individuals with diminishing TSP. These results suggest that pain-related fears mediate a greater proportion of the total effects of pain intensity on pain-related interference at higher levels of pain sensitivity compared with lower levels. Conceptually, we interpret these results to indicate that during activities, individuals with LBP who fall on the higher end of the spectrum of TSP may experience increases in their pain intensity over time compared with those LBP individuals on the lower end of the TSP spectrum. We suggest that incremental changes in pain intensity during activities may be a salient feature that evokes learning processes that influence which environments, activities and situations are associated with pain. The salience of these changes, however, is dependent on the individual's thoughts and beliefs around pain.

For example, within the chronic LBP population there are several pain-related behaviour phenotypes, including task avoidance, task persistence, pain avoidance and pain persistence. If we consider an activity such as ambulation, there will be individual differences in the amount of pain first felt as gait is initiated. There will also be individual differences in the how the nervous system endogenously modulates the input over time as the individual continues to ambulate. We suggest that individual differences in their ability to modulate pain may be reflective in their TSP score. Like the initial amount of pain felt during gait, increases or no change in pain intensity may signal harm or danger and evoke associative learning processes, whereas decreases in pain intensity over time may not. Changes in pain intensity during an activity over time also need to be cognitively evaluated, and depending on the individual's thoughts and feelings about pain, will have differential effects on the painrelated behaviour. A practical application of this hypothesis would be to assess the ability of TSP to discriminate pain-related behaviour phenotypes.

Our lack of main effects for TSP are contrary to the findings of George et al., 2006, who reported TSP explained additional variance in disability above and beyond pain-related fears (measured with FABQ-W) in a sample of patients with chronic low back pain (George et al., 2006). It is possible that the different sample populations between the studies could explain this discrepancy. In the current study, the sample consisted of individuals recruited from the general population with non-specific LBP, whereas the George et al., 2006 sample consisted of consecutive patients 
seeking care with chronic pain from a specialized tertiary care rehabilitation setting (George et al., 2006). These patients showed elevated mean pain-related disability, ODI $[17.30 \%(\mathrm{SD}=11.75)$ versus $52.6 \%$ $(\mathrm{SD}=13.0)]$, and FABQ-W scores [9.8 $(\mathrm{SD}=7.5)$ versus $31.5(\mathrm{SD}=11.2)$ ] compared with the group of participants in our current study (George et al., 2006). Differences in clinical pain intensity could not be assessed because of different assessment strategies. Comparing the two samples, it is possible that range of disability, clinical pain intensity and mediating factors is restrictive in our sample. Alternatively, our results indicate that the relationships among these factors are present but less direct in individuals with LBP who are not currently seeking interventions for their pain.

The current study has limitations and further research is needed in this area. First, our study design, which collected data at a single time point, (i.e., baseline measurements prior to receiving an intervention) does not allow for true temporal associations to be tested and the direction of effects was theoretically driven by the assumptions of the adopted FA model. Without collecting the data over time and testing temporal associations, caution is needed before making causal inference. Second, recent evidence has emerged which challenge the direction of effects adopted from the FA model. The FA model, like our study, assumes the effect of pain intensity of pain behaviour is mediated by fear. However, evidence from an experimental model showed that pain-related fear mediated differences in perceived pain intensity (Meulders et al., 2012). Further, two additional studies have shown that pain-related psychological measures of anxiety and fear mediated within person changes, and sex differences in pain sensitivity (Rhudy and Meagher, 2000; Horn et al., 2014). Thus, further model development and testing is needed to fully understand the direction of effects and the impact of these variables on one another. Third, regression casewise diagnostics found one case where the prediction error was 3 standard deviations from the mean error. Removal of this case did not alter the results. Additional regression diagnostic procedures identified approximately four cases that when dropped changed the results of the stage 1 and stage 2 moderated mediation regressions, where the addition of the interaction term did not provide significant improvement of the model (non-significant $\Delta R^{2}$ ). Inspection of these cases found their responses for pain intensity, pain-related fears and pain-related interference to be high, but reasonable for subjects with LBP to report. Thus, the unusualness of these cases may be a by-product of the sample's restricted range of pain-related disability.
The current cohort was recruited from the general population and as such reported much less painrelated disability than in cohorts recruited from clinical populations. To address this issue, similar statistical methods should be employed in larger cohorts that include additional subjects with higher levels of LBPrelated symptoms.

Despite these limitations, our results provide preliminary evidence that supports further investigations into how different pain modulatory tests interact with psychological variables to influence clinical measures of pain and disability. Our findings suggest interactions exist between pain-related fears and TSP that improve the predictive ability of the FA model in understanding the relationship between LBP intensity and interference in individuals with LBP who are not seeking treatment. Further elucidating the nature of these complex interactions using both cross-sectional and longitudinal data may provide new impetus for constructing more encompassing models and improving management of patients with LBP.

\section{Author contributions}

C.W.G. and M.D.B. contributed to the concept of the paper; J.E.B. and M.E.H. were involved in data acquisition; C.W.G. performed the analyses and drafted the article; C.W.G., M.E.H., M.D.B., M.E.R. and J.E.B. were instrumental in the interpretation of the models, critical revisions and final approval of the manuscript.

\section{References}

Alappattu, M.J., Bishop, M.D., Bialosky, J.E., George, S.Z., Robinson, M.E. (2011). Stability of behavioral estimates of activity-dependent modulation of pain. J Pain Res 4, 151-157.

Alschuler, K.N., Hoodin, F., Murphy, S.L., Rice, J., Geisser, M.E. (2011). Factors contributing to physical activity in a chronic low back pain clinical sample: A comprehensive analysis using continuous ambulatory monitoring. Pain 152, 2521-2527.

Anderson, R.J., Craggs, J.G., Bialosky, J.E., Bishop, M.D., George, S.Z., Staud, R., Robinson, M.E. (2013). Temporal summation of second pain: Variability in responses to a fixed protocol. Eur J Pain 17, 67-74.

Arendt-Nielsen, L., Yarnitsky, D. (2009). Experimental and clinical applications of quantitative sensory testing applied to skin, muscles and viscera. J Pain 10, 556-572.

Baron, R.M., Kenny, D.A. (1986). The moderator-mediator variable distinction in social psychological research: Conceptual, strategic, and statistical considerations. J Pers Soc Psychol 51, 1173-1182.

Bialosky, J.E., Bishop, M.D., Robinson, M.E., Zeppieri, G. Jr., George, S.Z. (2009). Spinal manipulative therapy has an immediate effect on thermal pain sensitivity in people with low back pain: A randomized controlled trial. Phys Ther 89, 1292-1303.

Bialosky, J.E., George, S.Z., Horn, M.E., Price, D.D., Roland, S., Robinson, M.E. (2014). Spinal manipulative therapy specific changes in pain sensitivity in individuals with low back pain (NCT01168999). J Pain 15, 136-148.

Bishop, M.D., Beneciuk, J.M., George, S.Z. (2011). Immediate reduction in temporal sensory summation after thoracic spinal manipulation. Spine J 11, 440-446. 
Brown, J.L., Edwards, P.S., Atchison, J.W., Lafayette-Lucey, A., Wittmer, V.T., Robinson, M.E. (2008). Defining patient-centered, multidimensional success criteria for treatment of chronic spine pain. Pain Med 9, 851-862.

Butsch, R.L.C. (1994). A workl sheet for the Johnson-Neyman technique. J Ecperimental Educ 12, 226-241.

Chapman, J.R., Norvell, D.C., Hermsmeyer, J.T., Bransford, R.J., DeVine, J., McGirt, M.J., Lee, M.J. (2011). Evaluating common outcomes for measuring treatment success for chronic low back pain. Spine (Phila $\mathrm{Pa}$ 1976) 36, S54-S68.

Chou, R., Qaseem, A., Owens, D.K., Shekelle, P. (2011). Diagnostic imaging for low back pain: Advice for high-value health care from the American College of Physicians. Ann Intern Med 154, 181-189.

Crombez, G., Eccleston, C., Van Damme, S., Vlaeyen, J.W., Karoly, P. (2012). Fear-avoidance model of chronic pain: The next generation. Clin J Pain 28, 475-483.

Deyo, R.A., Mirza, S.K., Martin, B.I. (2006). Back pain prevalence and visit rates: estimates from U.S. national surveys, 2002. Spine (Phila $\mathrm{Pa}$ 1976) 31, 2724-2727.

Dworkin, R.H., Turk, D.C., Wyrwich, K.W., Beaton, D., Cleeland, C.S., Farrar, J.T., Haythornthwaite, J.A., Jensen, M.P., Kerns, R.D., Ader, D.N., Brandenburg, N., Burke, L.B., Cella, D., Chandler, J., Cowan, P., Dimitrova, R., Dionne, R., Hertz, S., Jadad, A.R., Katz, N.P., Kehlet, H., Kramer, L.D., Manning, D.C., McCormick, C., McDermott, M.P., McQuay, H.J., Patel, S., Porter, L., Quessy, S., Rappaport, B.A., Rauschkolb, C., Revicki, D.A., Rothman, M., Schmader, K.E., Stacey, B.R., Stauffer, J.W., von Stein, T., White, R.E., Witter, J., Zavisic, S. (2008). Interpreting the clinical importance of treatment outcomes in chronic pain clinical trials: IMMPACT recommendations. J Pain 9, 105121.

Fairbank, J.C., Couper, J., Davies, J.B., O’Brien, J.P. (1980). The Oswestry low back pain disability questionnaire. Physiotherapy 66, 271273.

Fairbank, J.C., Pynsent, P.B. (2000). The Oswestry Disability Index. Spine (Phila Pa 1976) 25, 2940-2952, discussion 2952.

Fritz, J.M., George, S.Z., Delitto, A. (2001). The role of fear-avoidance beliefs in acute low back pain: Relationships with current and future disability and work status. Pain 94, 7-15.

George, S.Z., Fritz, J.M., Childs, J.D. (2008). Investigation of elevated fear-avoidance beliefs for patients with low back pain: A secondary analysis involving patients enrolled in physical therapy clinical trials. $J$ Orthop Sports Phys Ther 38, 50-58.

George, S.Z., Hirsh, A.T. (2009). Psychologic influence on experimental pain sensitivity and clinical pain intensity for patients with shoulder pain. J Pain 10, 293-299.

George, S.Z., Valencia, C., Zeppieri, G. Jr., Robinson, M.E. (2009). Development of a self-report measure of fearful activities for patients with low back pain: The fear of daily activities questionnaire. Phys Ther 89 , 969-979.

George, S.Z., Wittmer, V.T., Fillingim, R.B., Robinson, M.E. (2006). Fearavoidance beliefs and temporal summation of evoked thermal pain influence self-report of disability in patients with chronic low back pain. J Occup Rehabil 16, 95-108.

Gheldof, E.L., Crombez, G., Van den Bussche, E., Vinck, J., Van Nieuwenhuyse, A., Moens, G., Mairiaux, P., Vlaeyen, J.W. (2010). Pain-related fear predicts disability, but not pain severity: A path analytic approach of the fear-avoidance model. Eur J Pain 14, e871e879.

Hayes, A.F., Matthes, J. (2009). Computational procedures for probing interactions in OLS and logistic regression: SPSS and SAS implementations. Behav Res Methods 41, 924-936.

Horn, M.E., Alappattu, M.J., Gay, C.W., Bishop, M.D. (2014). Fear of severe pain mediates sex differences in pain sensitivity responses to thermal stimuli. Pain Res Treat 2014, 897-953.

Hubscher, M., Moloney, N., Leaver, A., Rebbeck, T., McAuley, J.H., Refshauge, K.M. (2013). Relationship between quantitative sensory testing and pain or disability in people with spinal pain - A systematic review and meta-analysis. Pain 154, 1497-1504.
IOM (Institute of Medicine) (2011). Relieving Pain in America: A Blueprint for Transforming Prevention, Care, Education, and Research (Washington, DC: The National Academies Press).

Latremoliere, A., Woolf, C.J. (2009). Central sensitization: A generator of pain hypersensitivity by central neural plasticity. J Pain 10, 895-926.

Leeuw, M., Goossens, M.E., Linton, S.J., Crombez, G., Boersma, K., Vlaeyen, J.W. (2007). The fear-avoidance model of musculoskeletal pain: Current state of scientific evidence. J Behav Med 30, 77-94.

Meulders, A., Vansteenwegen, D., Vlaeyen, J.W. (2012). Women, but not men, report increasingly more pain during repeated (un)predictable painful electrocutaneous stimulation: Evidence for mediation by fear of pain. Pain 153, 1030-1041.

Neziri, A.Y., Curatolo, M., Limacher, A., Nuesch, E., Radanov, B., Andersen, O.K., Arendt-Nielsen, L., Juni, P. (2012). Ranking of parameters of pain hypersensitivity according to their discriminative ability in chronic low back pain. Pain 153, 2083-2091.

Parr, J.J., Borsa, P.A., Fillingim, R.B., Tillman, M.D., Manini, T.M., Gregory, C.M., George, S.Z. (2012). Pain-related fear and catastrophizing predict pain intensity and disability independently using an induced muscle injury model. J Pain 13, 370-378.

Preacher, K.J., Hayes, A.F. (2004). SPSS and SAS procedures for estimating indirect effects in simple mediation models. Behav Res Methods Instrum Comput 36, 717-731.

Preacher, K.J., Rucker, D.D., Hayes, A.F. (2007). Addressing moderated mediation hypotheses: Theory, methods, and prescriptions. Multivariate Behav Res 42, 185-227.

Rhudy, J.L., Meagher, M.W. (2000). Fear and anxiety: Divergent effects on human pain thresholds. Pain 84, 65-75.

Roland, M., Fairbank, J. (2000). The Roland-Morris disability questionnaire and the Oswestry disability questionnaire. Spine (Phila Pa 1976) 25, 3115-3124.

Sarkar, S., Woolf, C.J., Hobson, A.R., Thompson, D.G., Aziz, Q. (2006) Perceptual wind-up in the human oesophagus is enhanced by central sensitisation. Gut 55, 920-925.

Staud, R., Price, D.D., Fillingim, R.B. (2006). Advanced continuouscontact heat pulse design for efficient temporal summation of second pain (windup). J Pain 7, 575-582.

Staud, R., Weyl, E.E., Price, D.D., Robinson, M.E. (2012). Mechanical and heat hyperalgesia highly predict clinical pain intensity in patients with chronic musculoskeletal pain syndromes. J Pain 13, 725-735.

Sullivan, M.J.L., Bishop, S.R., Pivik, J. (1995). The pain catastrophizing scale: Development and validation. Psychol Assessement 7, 524-532.

Todd, A.J. (2010). Neuronal circuitry for pain processing in the dorsal horn. Nat Rev Neurosci 11, 823-836.

Valencia, C., Fillingim, R.B., George, S.Z. (2011). Suprathreshold heat pain response is associated with clinical pain intensity for patients with shoulder pain. J Pain 12, 133-140.

Vlaeyen, J.W., Linton, S.J. (2012). Fear-avoidance model of chronic musculoskeletal pain: 12 years on. Pain 153, 1144-1147.

Von Korff, M., Ormel, J., Keefe, F.J., Dworkin, S.F. (1992). Grading the severity of chronic pain. Pain 50, 133-149.

Waddell, G., Newton, M., Henderson, I., Somerville, D., Main, C.J. (1993). A Fear-Avoidance Beliefs Questionnaire (FABQ) and the role of fear-avoidance beliefs in chronic low back pain and disability. Pain 52, 157-168.

Walker, B.F. (2000). The prevalence of low back pain: A systematic review of the literature from 1966 to 1998. J Spinal Disord 13, 205-217.

Zhou, Q., Price, D.D., Callam, C.S., Woodruff, M.A., Verne, G.N. (2011). Effects of the N-methyl-D-aspartate receptor on temporal summation of second pain (wind-up) in irritable bowel syndrome. J Pain 12, 297-303.

\section{Web reference}

Hayes, A.F. (2012) PROCESS: A versatile computational tool for observed variable mediation, moderation and conditional process modeling [White Paper]. Retrieved from http://www.afhayes.com/public/ process2012.pdf (accessed 1 December 2012). 